\title{
Abridged Shape Matrix Representation for the Recognition of Aircraft Targets from 2D ISAR Imagery
}

\author{
Hari Kishan Kondaveeti $^{1^{*}}$ and Valli Kumari Vatsavayi ${ }^{2}$ \\ ${ }^{1}$ Department of CS \& SE, AUCE (A), Andhra University, Visakhapatnam, India. \\ ${ }^{2}$ Department of CS \& SE, AUCE (A), Andhra University, Visakhapatnam, India.
}

\begin{abstract}
Inverse Synthetic Aperture Radar (ISAR) images are used (i) for detecting the high scatter parts or areas in the target (hot-spots) and (ii) for the identification of targets. Automatic Target Recognition (ATR) systems reduce the burden of the human operators by identifying the targets in ISAR images automatically and aid the operators in executing accurate decisions with less effort. Even though target classification or recognition is the main objective these systems, they must possess the mechanisms to cope up with the noise as the clutter and noise degrade the overall performance of these systems significantly.

In this paper a new classification method is proposed to identify the aircrafts in ISAR images automatically. A Homomorphic wavelet based step-by-step systematic noise removal procedure is developed, to segment out the target carefully from background noise. Rotation and scale normalization are performed on the segmented image using feature matching. Finally, the classification step depends on the abridged shape matrix generated from the segmented target to recognize the aircraft. Experiments were conducted on synISAR aircraft image dataset and results are presented. The experimental results show that the proposed method is fast and accurate to identify aircrafts in ISAR images.
\end{abstract}




\section{INTRODUCTION}

Inverse Synthetic Aperture Radar (ISAR) is a radar, analogous to conventional Synthetic Aperture Radar (SAR), used to generate two dimensional (2D) high resolution radar images (ISAR images) of the targets such as ships, aircrafts and space objects moving in a non-cooperative way. ISAR sends directional pulses of electromagnetic energy and analyses the energy reflected from the object back to the radar station to detect the presence, position and motion of a target (Scanning phase) and maps the scattering coefficients onto a two-dimensional plane (Image generation phase). This two dimensional representation of the ISAR output carries the 2D distribution of the electromagnetic energy backscattered from the target. ISAR radars are used in maritime patrol to generate ISAR images for identification and classification of targets in different weather conditions like snow fall, rain, fog and mist, and day and night. Generally, human operators are trained to process the ISAR images and detect the targets. They are required to distinguish a large number of targets such as ships and aircrafts from ISAR images. The identification process requires the operators to dwell for a relatively long time duration before an identification can be confirmed. Manual surveillance is difficult, time consuming, laborious and tedious. Automatic Target Recognition System (ATR) reduces the burden of the human operators by processing and classifying the ISAR images automatically. Even though extensive research work has been going on to develop ATRs, the problem of automatic identification of targets using ISAR images is still a challenging because of following reasons. (i) ISAR images are quite different from optical images, (ii) ISAR images may not contain the sufficient information for target recognition, (iii) Poor resolution, Low Signal to Noise Ratio (SNR), Blur may be associated with ISAR images, (iv) Clutter and noise may exist, (v) Different targets may have similar shape in ISAR images etc. These problems degrade the overall performance of the ATR system significantly. In the literature of ISAR image classification, it is found that the noise removal problem in ISAR images is addressed using spatial image enhancement techniques. Some researchers [1], [7] used global threshold based noise removal techniques to remove the noise. Some researchers [9], [14] used spatial filters and morphological techniques to address the noise. Global threshold based noise removal methods used in [1], [7] mainly depends on the threshold selected for the noise removal. The pixel intensities above the global threshold are changed to zero while keeping the pixels unmodified having intensity levels below the threshold. Obtaining the optimal global threshold value is the main drawback in these methods. Image enhancement techniques described in [9], [14] focussed on spatial domain, which are less effective. The noise present in radar images is speckle noise, which is multiplicative. The speckle noise is difficult to model because the signal and speckle noise are statistically does not depend on each other and it cannot be removed simply by subtracting the noise from the image because the noise is not additive. In the proposed method, the noise is addressed in 
frequency domain for efficient noise removal. Wavelets are the promising approach for the removal of the speckle noise efficiently. Homomorphic Wavelet based filtering procedure is developed in this paper to remove the noise. Some additional image enhancement operations are applied to segment out the target from the remaining noise and clutter.

2D ISAR image based target classification can be found in [1], [2], [6], [3], [7], [4], [8] and [5]. The methods described in [1], [2], [3] and [4] mainly deal with classification of ship targets. They cannot be applied directly to other types of targets like aircrafts because they mainly depend on the 2D and 3D models of the ships. [8], [6], [5], and [7] deal with the aircraft classification. Botha [8] used four types of features namely geometrical moments, shape features, invariant features based on moments and quantized energy features, and used nearest neighbour classifier and feed forward neural nets as classifiers. In [6] an approach based on Zernike moments, has been described to guarantee scale and rotation invariance of ISAR images. Kim et al. [7] described a new approach for the classification of aircrafts, which relies on feature vectors generated from image itself, rather than considering geometric, statistical characteristics. Saidi et al. [5] fused two shape descriptors, moment invariants and Fourier descriptors to generate feature vector.

In the classification of ISAR images, the main difficulty originates from two issues. They are (i) the dimensionality of feature vector and (ii) the discriminativeness of the features, extracted from an ISAR image. High dimensional features are more discriminative as they carry more target information, but they introduce computational complexity. Lower dimensional features are less discriminative but classification time is very less. Highly discriminative low dimensional features are ideal features and are most desirable as they reduce the computational complexity without affecting the accuracy of the classifier. It is, however, difficult to extract this type of features and we have to compromise either at computational complexity or at accuracy of the classifier.

The methods described in [8], [5] and [6] are based upon the low dimensional local and geometric characteristics of an ISAR image. They tried to simplify the classifier structure in order to save the computational resources. But, as explained above these features are less discriminative and may not be successful always. The method proposed in [7] extracts the lower dimensional feature vectors from image represented in polar form and reduces the storage space drastically. However, the computational complexity of this method is high due to the complex structure of the classification procedure. The pre-processing method used in [7] is simple and trivial, whereas the reduction of noise in real ISAR images is difficult and not straight-forward. Representing the ISAR images in Polar coordinate system and extracting feature vectors is computationally expensive and accuracy of the classifier depends on the selection of optimal threshold values in coarse search and fine search steps. 
The method proposed in [4], extracts the Co-occurrence Histogram Oriented Gradient (CoHOG) features which are high dimensional. To handle the high dimensionality of features, Random Ensemble Metric (REMetric) is used to calculate the similarity between two feature vectors. It is highly accurate method and robust against selfocclusions of targets. As it uses multiple support vector machines (SVM's), the computational complexity is slightly high.

Statistical measures based representation methods discussed above, represent the targets using area, centroid, variance, skewness and moments etc. Shapes based methods represent the target in the form of numerical coding or outline description. Shape based descriptors are global descriptors, they do not consider the inner details of the targets which play important role in distinguishing two targets of nearly similar shape. The local descriptor methods are not used generally for the ISAR image classification, because the classifier may fail due to the abnormal changes in the local features due to scaling, rotation and flaws in signal extraction.

The abridged shape matrix described in this paper is not only based on global geometrical shape, it also represents the local features of a target and these features are scale, rotation and translation invariant. Instead of extracting features using different feature extraction methods, the proposed method depends on the low dimensional, concise representation of the original pixel intensity values of the image itself.

In this paper, the work is aimed at the noise removal and the target extraction from ISAR images; and classification of the extracted targets. Our contributions in this work are: First one is the development of wavelet based noise filtering approach and the second one is the development of abridged shape matrix model for target representation which is robust, concise and non-redundant.

There are some other issues addressed in this paper like reduction of the execution time and the problem of insufficient size of data available for training simultaneously while making the recognition rate of the classifier good. A brief discussion on solving these issues is presented here.

The accuracy of the classifier depends on the size of the training database used to build a classifier model. Some times in real scenarios, however, sufficient number of input images may not be available for training. Then the design of the classifier must be according to the size of the training database available. In the proposed method, the classifier is designed for the worst case scenario, having the size of the training database very low consisting only one ISAR image per a given aircraft.

The execution time of a classifier depends on complexity of its structure, dimensionality of the features, search space, number of phases involved in making a final decision and threshold values to be selected in each phase [7]. Even though the size of the generated feature vector is small, if the final decision of the classifier 
depends on the threshold values selected at each phase. The accuracy of the classifier is biased in selecting optimal threshold values. Finding the optimal threshold values becomes another research problem here. In order to reduce the recognition time, the proposed method focussed on reduction of the classifier complexity by reducing the number of phases involved in classifier decision and did not use any threshold based mechanisms. The search space is very low due to the size of the trained data is very less having one image per an aircraft. The detailed description of the proposed method is discussed in remaining sections.

In the remainder of this paper, the proposed classification algorithm is presented in section II. Section III contains classification experiments, performance of proposed method on synISAR image database and associated discussions. The paper is finally concluded in Section IV.

\section{P ROPOSED CLASSIFICATION METHOD}

\section{A. Pre-processing}

Noise degrades the quality of the ISAR images, which further reduces the overall accuracy of the Automatic Recognition Systems. To improve the accuracy of the classification process, suppression of noise and stripes must be done before extraction of the features from images.

1) Reduction of Speckle noise: A combination of Homomorphic filtering and Wavelet filtering techniques is applied for the reduction of speckle noise [13]. Initially, the Homomorphic filter is used to transform the image into log domain, and then a Wavelet filter is applied to eliminate certain frequencies.

Speckle, a high frequency component, is a granular noise that inherently exists in and degrades the quality of the ISAR images. Speckle noise in ISAR images is considered serious because it causes difficulties for image interpretation.

Speckle noise present in radar images is considered as multiplicative noise. Multiplicative noise is the result of the multiplication of two signals, and is in direct proportion to the local grey level in any area [15]. The following model is considered a good model for images with speckle noise:

$$
\mathrm{I}^{\prime}(\mathrm{x}, \mathrm{y})=\mathrm{I}(\mathrm{x}, \mathrm{y}) \eta(\mathrm{x}, \mathrm{y})
$$

Where $I^{\prime}$ is, the resultant of the image I corrupted with noise $\eta$

Homomorphic filtering technique is used for removing multiplicative noise. This filter converts the multiplicative noise to additive, as many noise reduction filters are created to eliminate random noise which occurs 
additively. Speckle noise is converted to additive by applying logarithmic transform on the original image as shown in the following equation.

$$
\log I^{\prime}(x, y)=\log I(x, y)+\log \eta(x, y)
$$

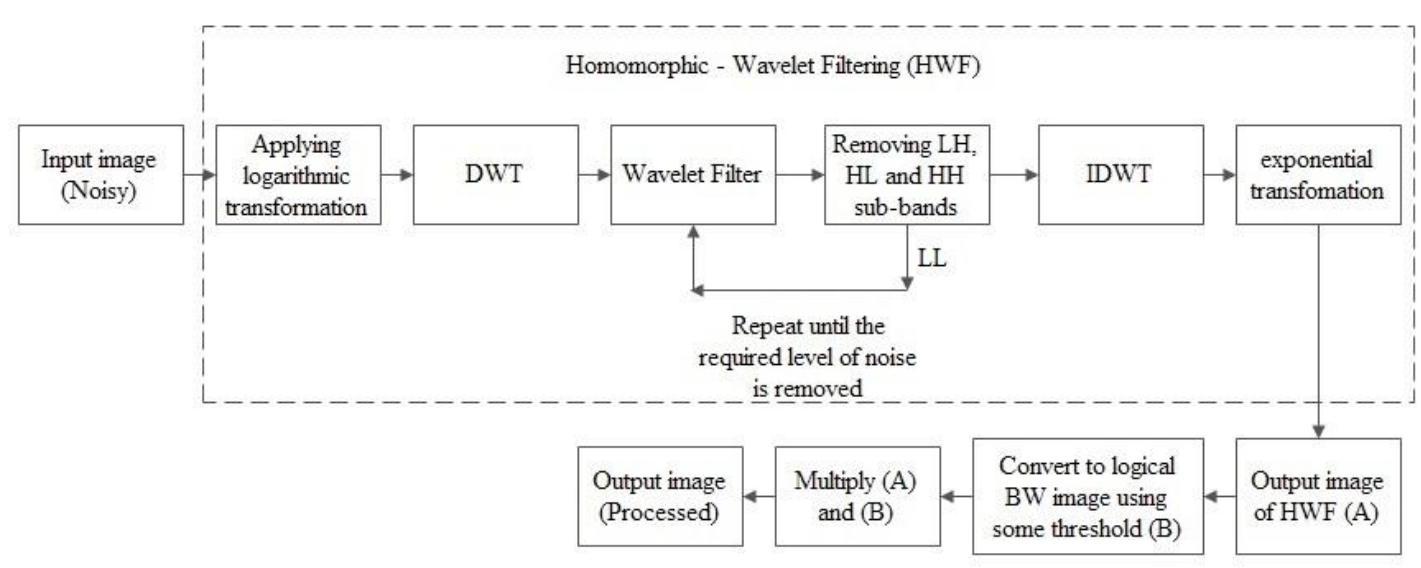

Fig. 1. Overall description of pre-processing method

After the logarithmic transformation, Discrete Wavelet Transform (DWT) is applied on the image. DWT decomposes the image into four sub-band images HH, LH, HL and LL. LL image represents smoothing property, and contains the low frequencies of the image which contribute the global description of the image. $\mathrm{LH}, \mathrm{HL}$ and $\mathrm{HH}$ images represent the intensity variations of the image along vertical, horizontal and diagonal directions respectively, and contain most of the images high frequencies and noise. The noise is removed by eliminating the high frequency sub band images $\mathrm{HH}, \mathrm{HL}$ and $\mathrm{HH}$ by keeping the sub band image LL alone. The elimination of these images, does not affect the accuracy of classifier, because LL holds the crucial information required for the target recognition. Even if this LL sub-band image contains noise, pass this image as input to the Homomorphic wavelet filter again. This process may be continued iteratively, until the noise is eliminated. However, due to removing high-frequency bands iteratively, some crucial details of the image may also be lost.

Level-2 was found to be optimal filtering level for removing speckle noise present in our ISAR images, where Homomorphic filter is applied twice. Figure 2 (b) gives the LL sub band image obtained by applying Homomorphic wavelet filter twice on image in figure 2 (a). This image in figure 2 (b) has to be further processed to eliminate the remaining noise. 
2) Elimination of Outliers: To remove the details, other than the target in the above processed image, the following procedure is used: First, the image is converted to black and white (logical image) by using a selected threshold, the result is shown in Figure 2 (c). Next, the images in (b) and (c) are multiplied. Finally, the Homomorphic filtered image, figure 2 (d), is obtained having no noisy details around the target. This processed image is passed to the feature extraction module.

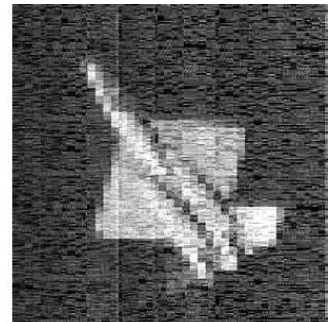

(a)

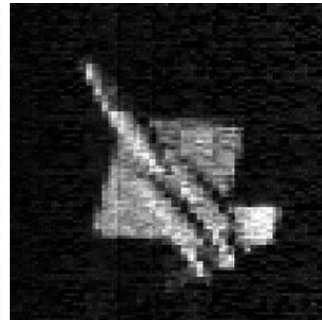

(b)

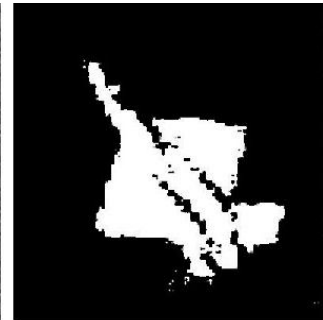

(c)

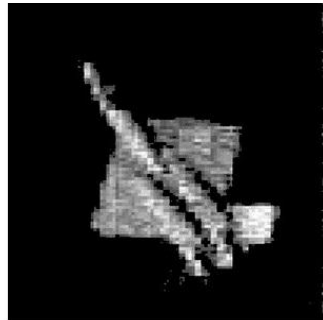

(d)

Fig. 2. Steps involved in Preprocessing of ISAR image of F15 fighter jet at aspect angle 35. (a) Noisy image considered for Pre-Processing; (b)Removing of sub-bands LH, HL, HH from the noisy image by the application of Homomorphic Wavelet Filter at level 2; (c)Converting the filtered image to Black and White image, using a threshold 0.2; (d) Result of multiplying the filtered image (b) with the black and white image (c)

\section{B. Scale and Rotation normalization}

After the pre-processing, scale and rotation normalization are performed on the test image. However, there is no need to perform the scale and rotation normalization on the test image because the abridged shape matrix obtained from the test image is a scale, rotation and translation invariant. But the problem occurs with the size of the trained database. More number of training images are needed for training to capture the variations correctly using shape matrices. In the proposed method, the lack of sufficient database for training is also considered as a problem to be addressed. In order to cope up with the insufficient size of the training database and for generating robust shape descriptor, sale and rotation normalization are performed on the processed test image. The normalization is performed using feature matching technique. SURF [16] features are extracted from the test image and trained images. The extracted features are matched and locations of corresponding points are retrieved for each image. Then the geometric transformation between the two images is calculated [12] and the test image is rotated and scaled to the orientation and size of the trained image. 


\section{Abridged Shape Matrix representation}

Shape matrix [18], [17] is used to describe and discriminate the planar objects in binary images. The Shape descriptor is a matrix, known as Shape matrix, that represents the shape of an object. The shape matrix is obtained by the polar quantization of both the shape outline and inner details of the object. The shape matrix descriptor is independent of object's scaling, rotation and translation.

The procedure of obtaining the shape matrix [17] of size $\mathrm{m} \times \mathrm{n}$ is presented here briefly. Initially, center of gravity 0 of the binary object is obtained. Then the distances of the boundary pixels from the center of gravity are calculated. The maximum distance value $\mathrm{D}$ is considered as the radius of the circle in which the object can completely lie in. $\mathrm{OR}$ is the line joining the center of gravity $\mathrm{O}$ and the maximum distance pixel $\mathrm{R}$ on the border of the given object and the length of the line joining OR is $\mathrm{D}$. Then the line joining $\mathrm{OR}$ is divided into $\mathrm{n}-1$ equal lengths. Concentric circles with the radii $D /(n-1), 2 D /(n-1), \ldots(n-2) D /(n-1),(n-1) D /(n-1)$ are drawn as the center of gravity 0 as the center. $m$ number of radial lines which divide the concentric circles into $\mathrm{m}$ equal arcs are drawn with an angle $(360 / \mathrm{m})^{0}$ starting from OR. A shape matrix of size $\mathrm{m} \times \mathrm{n}$ can be obtained by sampling $\mathrm{m} \times \mathrm{n}$ pixels from the intersection points of the $m$ radial lines and $n$ concentric circles. The elements of the shape matrix are filled with 1's if a sampled point lies inside the shape otherwise filled with 0 's. Figure 3 depicts the shape matrix of size $10 \times 4$ obtained from polar quantization of an aircraft.
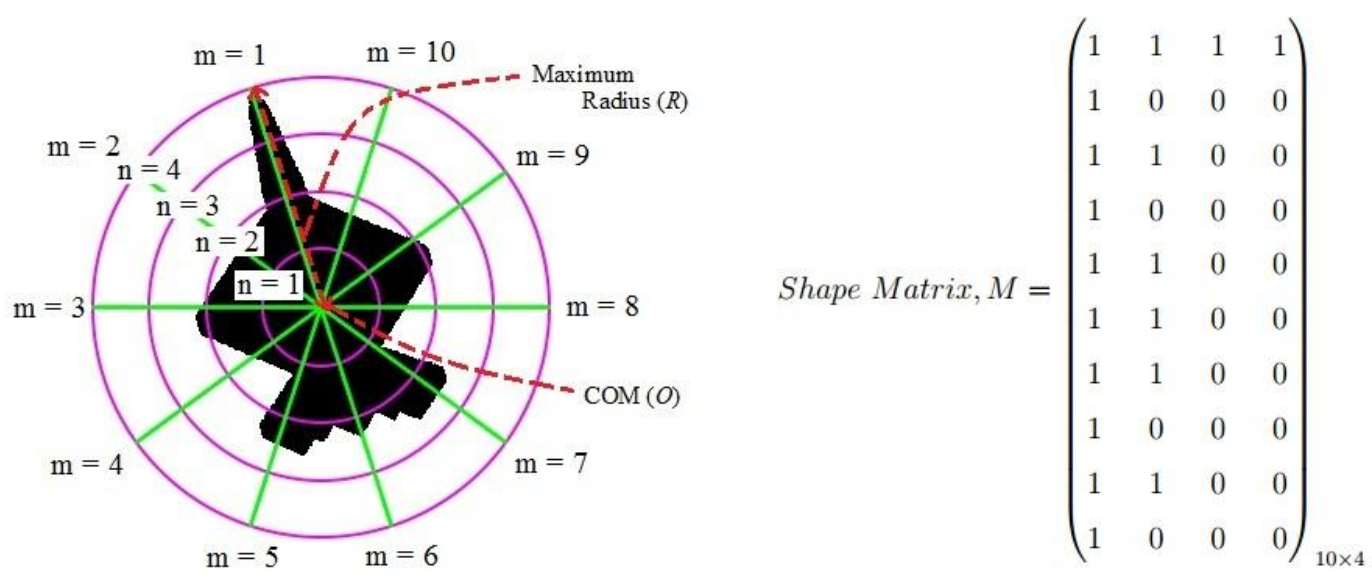

Fig. 3. Polar Quantization of aircraft image. (a) Polar grid over the aircraft image having no. of radial lines $(m)=10$ and no. of concentric circles $(n)=4$; (b) Shape matrix derived from the aircraft having no. of rows $(\mathrm{m})=10$ and no. of columns (n) $=4$. 
The similarity between two targets can be found using the formula given in equation (3) defined in [17].

$$
\text { Similarity }=1-[\mathrm{S} / \mathrm{mn}]
$$

Here $\mathrm{S}$ is obtained by performing exclusive $\mathrm{OR}(\mathrm{XOR})$ operation between the two shape matrices obtained from the targets present in the input images. The similarity value lies between 0 and 1 . If the similarity value is close to the zero the two images are dissimilar and if the value approaches 1 the images are similar.

The main drawbacks of this method are (i) Accuracy of the classification using shape matrices depends upon the size of the shape matrix i.e number of samples in polar quantization, more the number of samples more the accuracy of the classification; (ii) Computational complexity increases with the number of samples considered in the polar quantization; (iii) The sampling density of the polar sampling raster is not constant; (iv) With the increasing size of the shape matrix more redundancy occurs in shape matrix representation due to over sampling in the inner circles nearer to the center of gravity; (v) Due to over sampling of the data more computational resources are wasted in interpolating redundant data; (vi) Shape matrix generated is based on the maximum radius of the binary shape. If the shape has more than one maximum radius, then a separate shape matrix has to be obtained for every maximum radius. The classification task becomes complex as the number of comparisons increases with the increase in the number of shape matrices per object.

A weighting scheme [18] was introduced to address the problem of redundancy in inner most shape samples. This scheme assigns weight to the samples obtained from polar quantization based upon the distance of the shape samples from the center of gravity. Outer most shape samples are assigned with maximum weight. The weight assigned is decreases gradually with the inner shape samples and becomes negligible (near to zero) with the inner most shape samples which are nearer to the center of gravity. The authors claimed that this method is more robust and it gives better results than the method described in [17] as they gave more preference to the non-redundant data by assigning more weight to it.

The limitations of this method are (i) Redundancy of the innermost samples is addressed but many of the limitations are left unaddressed; (ii) This method adds additional computational overheads by calculating and assigning weights to the samples.

As discussed above, method [17] spends computational resources unnecessarily in interpolating the redundant data repeatedly for the same shape samples. The weighting scheme [18] calculates and assigns negligible weights to the redundant data obtained. This method adds additional burden on the resources to add negligible weights. Instead of interpolating redundant data and assigning low weight to it, it is 
better to avoid interpolating the redundant data as it contributes very less information for the classification decision. This is the basic idea on which the abridged shape matrix representation proposed in this paper is built.

To overcome the flaws in the above methods, the abridged shape matrix representation is proposed in this paper. The abridged shape matrix is a concise representation of the shape matrix described in [17]. It avoids the unnecessary interpolations in representing redundant data and avoids the weighting scheme. Comparatively less computational resources are utilized for this method than the methods [17], [18] to compute the same information. The proposed method carefully avoids the interpolation of the over sampled data in innermost shape samples by checking the conditions derived in the proposed method that give the presence of over sampling. Even though innermost shape samples are not considered, the discriminative information is preserved for classification as this method considers outer most shape samples which are assigned more weight in the weighting method.

To explain the procedure of obtaining the abridged shape matrix, let us consider the general image representation in Cartesian coordinate system. Let $I(x, y)$ be a 2dimensional image and a pixel located at the coordinates $(x, y)$ in the image $I(x, y)$ is represented by an intensity value. Here $x$ and $y$ are two integers that represent the relative horizontal and vertical positions of a pixel. Generally all the pixel coordinates are represented as integers and the distance between two horizontal or vertical pixels is considered as one unit. Sometimes, images are converted between Cartesian to Polar and Polar to Cartesian coordinate systems as a part of processing. In a conversion process of Polar to Cartesian coordinate system, if the coordinate values obtained are real valued they are replaced by nearest integer values by rounding the values or by an interpolation technique. Interpolation adds some additional overhead in the conversion process.

1) Obtaining the size of shape matrix dynamically: As explained above, the length of the line joining OR (maximum radius) calculated by the Euclidean distance measure is $\mathrm{D}$ units i.e., the maximum number of pixels that lie on the line joining $\mathrm{OR}$ is equal to rounded value of $\mathrm{D}$ to the nearest integer. It is clear that maximum $\mathrm{D}$ samples can be taken from the line joining OR without over sampling. The $\mathrm{n}$ value, which specifies the number of the sampling points on a radial axis, chosen must be less than or equal to $\mathrm{D}$.

$\mathrm{D}=$ The length of the line joining OR round $(\mathrm{D})=$ The number of pixels lie on the line joining OR i.e maximum number of samples can be chosen without over sampling $\mathrm{n}=$ Number of sample points considered on the line joining OR for polar quantization 
Therefore, to avoid interpolation the $\mathrm{n}$ should be chosen as

$$
\mathrm{n} \leq \operatorname{round}(\mathrm{D})
$$

In the similar way, perimeter of the circle with $O R$ as the radius having length $D$ is $2 \pi \mathrm{D}$ units i.e., the maximum number of pixels that lie on the perimeter of the circle is equal to rounded value of $2 \pi \mathrm{D}$ to the nearest integer. Maximum $2 \pi \mathrm{D}$ samples can be taken from the perimeter without over sampling. The $\mathrm{m}$ value, which specifies the number of the sampling points on the perimeter of the circle, chosen must be less than or equal to $2 \pi \mathrm{D}$.

$2 \pi \mathrm{D}=$ The Perimeter of the circle with $\mathrm{OR}$ as radius having length $\mathrm{D}$

round $(2 \pi \mathrm{D})=$ The number of pixels lie on the perimeter of the circle i.e maximum number of samples can be chosen without over sampling

$\mathrm{m}=$ Number of sample points considered on the perimeter of the circle for polar quantization

Therefore, to avoid interpolation the $\mathrm{m}$ should be chosen as

$$
\mathrm{m} \leq \operatorname{round}(2 \pi \mathrm{D})
$$

2) Avoiding over-sampling: The data redundancy in the shape matrices obtained by the above methods [18], [17] is due to static size of shape matrices. Use of dynamic shape sizes for obtaining the shape matrices can reduce the over sampling problem up to some extent but it cannot reduce the problem completely. In most of the cases, over sampling may occur in the innermost shape samples even after using dynamic size for the shape matrix. Figure 4 shows the over sampling in inner shape samples for different $\mathrm{m}$ and $\mathrm{n}$ values chosen for polar quantization.

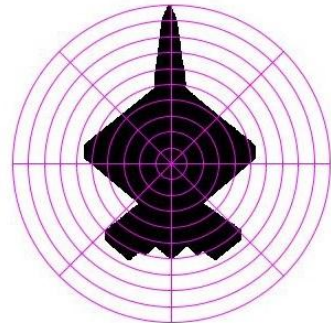

(a)

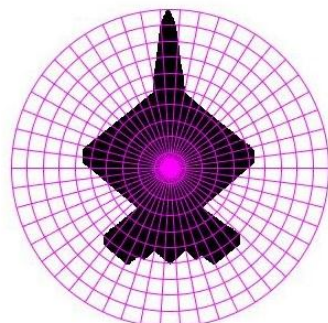

(b)

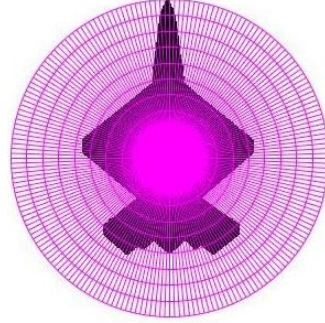

(c)

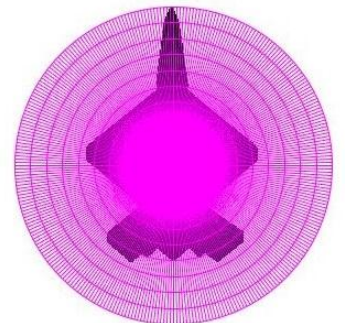

(d)

Fig. 4. Over sampling in inner most shape samples. (a) No over sampling when $\mathrm{m}=8$, $\mathrm{n}=10$; (b) Over sampling in innermost circle when $\mathrm{m}=50, \mathrm{n}=10$; (c) Over sampling in inner concentric circles 1,2 and 3 when $\mathrm{m}=200, \mathrm{n}=10$; (d) Over sampling in inner concentric circles 1,2,3 and 4 when $m=300, n=10$. 
To avoid the over sampling problem, initially $\mathrm{m}$ and $\mathrm{n}$ values are determined based on the conditions given in the equations (4) and (5). The perimeter of every concentric circle is calculated. Over sampling occurs if the number of radial lines $m$ which divides the concentric circles into $\mathrm{m}$ equal arcs is greater than the number of pixels on the perimeter the concentric circle. To reduce the above problem, shape abridged matrix is generated from the concentric circle whose perimeter is greater than number of radial lines $\mathrm{m}$ i.e rows of the shape matrix are sampled from this circle onwards. Figure 5 shows the over sampling in inner concentric circles 1,2 and 3 i.e., $\mathrm{n}=1,2$ and 3. There is no over sampling in outer concentric circles where $n=4$ to 10 .

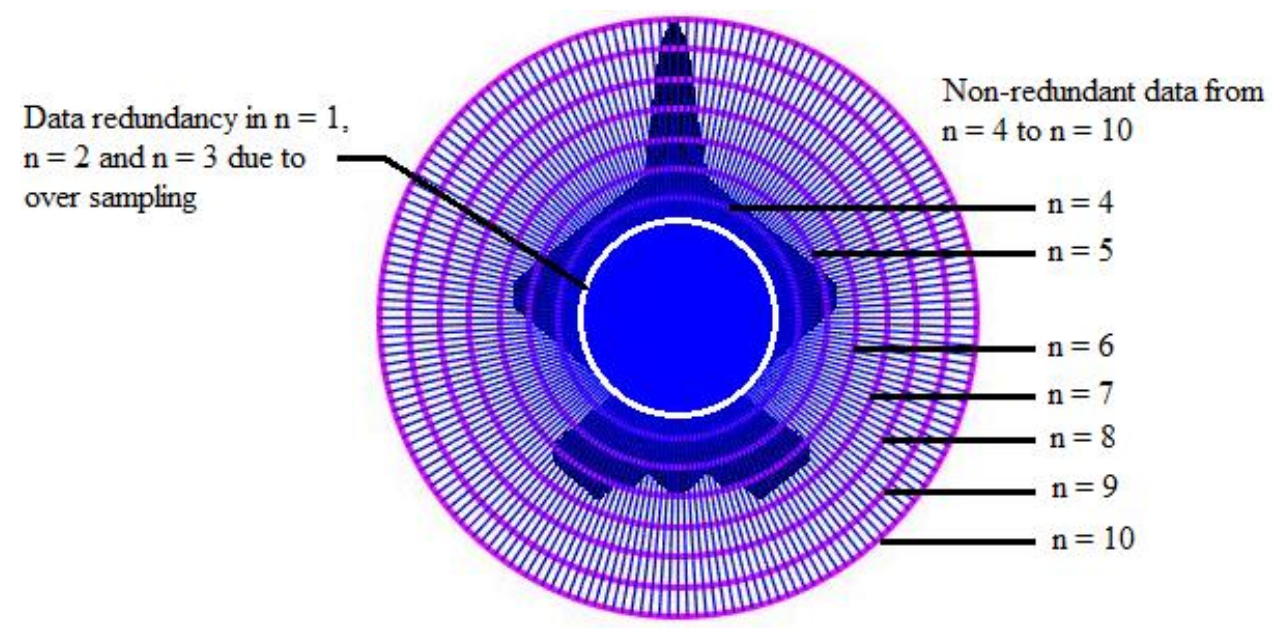

Fig. 5. Over sampling in innermost circles 1, 2 and 3 due to improper selection of $m$ and $\mathrm{n}$ values for the generation of shape matrix.

The method [17] generates a shape matrix of size $200 \times 10$ by considering the over sampled data also. But, the proposed method carefully obtains the abridged shape matrix of size $200 \times 7$ by avoiding unnecessary interpolations. Figure 6 shows the shape matrices obtained by the method [17] and the proposed method. These shape matrices are obtained from the aircraft image in figure 5.

In figure 6 , the shape matrix $\mathrm{M}$ is generated by sampling the data from all the intersection points of 200 radial lines and 10 concentric circles. The generation of shape matrix does not consider the over sampling problem. Over sampled data in innermost circles $n=1,2$ and 3 causes the data redundancy in the first three columns of the shape matrix. The generation process of abridged shape matrix automatically checks every concentric circle to verify the presence of over sampled data. After finding the concentric circle which is not affected by over sampling, the sampling process is started to generate the abridged shape matrix. Abridged shape matrix does not contain the redundant information as it leaves the over sampled data. The first 
column of the abridged shape matrix is obtained by sampling the data from $4^{\text {th }}$ concentric circle i.e $n=4$.

Instead of generating shape matrices based on static size defined initially by assigning some arbitrary values to $m$ and $n$ manually, the proposed method calculates the optimal $m$ and $n$ values dynamically based on the length of the maximum radius. Steps involved obtaining the abridged shape matrix are explained here.

1) Initially center of gravity $O$ of the given aircraft is obtained.

2) Maximum radius $D$ of the aircraft object is calculated.

3) The perimeter of the outer most circle is calculated as $P=2 \pi D$.

4) No. of radial lines $m$ is taken arbitrarily satisfying the condition given in the equation (5) preferably a value between $P / 2$ and $P$.

5) No. of concentric circles $n$ is taken arbitrarily satisfying the condition given in the equation (4) preferably a value between $D / 2$ and $D$.

6) The abridged shape matrix is generated from the concentric circle whose perimeter value is greater than $m$.

The generated abridged shape matrices are used to calculate the similarity between the images. The classification procedure is described in the following section.

\begin{tabular}{|c|c|c|c|c|c|c|c|c|}
\hline & & $\begin{array}{c}n=1 \\
\text { column-1 }\end{array}$ & $\begin{array}{c}n=2 \\
\text { column-2 }\end{array}$ & $\begin{array}{c}n=3 \\
\text { column-3 }\end{array}$ & $\cdots$ & $\cdots$ & $\begin{array}{c}n=9 \\
\text { column-9 }\end{array}$ & $\begin{array}{c}n=10 \\
\text { column-10 }\end{array}$ \\
\hline \multirow{6}{*}{ Shape matrix, $\mathrm{M}=$} & $m=1$ & $\Gamma a_{1,1}$ & $\mathrm{a}_{1,2}$ & $\mathrm{a}_{1,3}$ & $\ldots$ & $\ldots$ & $\mathrm{a}_{1,9}$ & $\left.\mathrm{a}_{1,10}\right]$ \\
\hline & $\mathrm{m}=2$ & $\mathrm{a}_{2,1}$ & $a_{2,2}$ & $a_{2,3}$ & $\ldots$ & $\ldots$ & $\mathrm{a}_{2,9}$ & $\mathrm{a}_{2,10}$ \\
\hline & : & : & : & : & $\ldots$ & $\cdots$ & : & : \\
\hline & : & : & : & : & $\cdots$ & $\cdots$ & : & : \\
\hline & $m=199$ & $a_{199,1}$ & $a_{199,2}$ & $a_{199,3}$ & $\ldots$ & $\ldots$ & $a_{199,9}$ & $\mathrm{a}_{199,10}$ \\
\hline & $\mathrm{m}=\mathbf{2 0 0}$ & $a_{200,1}$ & $a_{200.2}$ & $a_{200.3}$ & $\ldots$ & $\ldots$ & $a_{200.9}$ & $a_{200,10}$ \\
\hline
\end{tabular}

\begin{tabular}{|c|c|c|c|c|c|c|c|c|}
\hline & & $\begin{array}{c}n=4 \\
\text { column-1 }\end{array}$ & $\begin{array}{c}n=5 \\
\text { column-2 }\end{array}$ & $\begin{array}{c}n=6 \\
\text { column-3 }\end{array}$ & $\cdots$ & $\cdots$ & $\begin{array}{c}n=9 \\
\text { column-6 }\end{array}$ & $\begin{array}{c}\mathrm{n}=10 \\
\text { column- } 7\end{array}$ \\
\hline \multirow{6}{*}{ Abridged Shape Matrix, ASM = } & $m=1$ & {$\left[\mathrm{a}_{1,1}\right.$} & $\mathrm{a}_{1,2}$ & $\mathrm{a}_{1,3}$ & $\ldots$ & $\ldots$ & $\mathrm{a}_{1,6}$ & $\left.\mathrm{a}_{1,7}\right]$ \\
\hline & $\mathrm{m}=2$ & $a_{2,1}$ & $a_{2,2}$ & $a_{2,3}$ & $\ldots$ & $\ldots$ & $a_{2,6}$ & $a_{2,7}$ \\
\hline & : & : & : & : & $\ldots$ & $\ldots$ & : & : \\
\hline & : & : & : & : & $\ldots$ & $\ldots$ & : & : \\
\hline & $\mathrm{m}=199$ & $a_{199,1}$ & $a_{199,2}$ & $a_{199,3}$ & $\ldots$ & $\ldots$ & $a_{199,6}$ & $a_{199,7}$ \\
\hline & $\mathrm{m}=200$ & $a_{200,1}$ & $a_{200.2}$ & $a_{200.3}$ & ... & $\ldots$ & $a_{200.6}$ & $\left.\mathrm{a}_{200,7}\right]$ \\
\hline
\end{tabular}

Fig. 6. Shape matrices obtained using the method described in [17] and Proposed method. 


\section{Classification:}

After obtaining the abridged shape matrices from the above step, the similarity value is calculated between the shape matrices using the measure described in the section II.B in the equation (3). The maximum value of the similarity values calculated for the test image and given trained image is stored in a similarity measure vector along with the class label of the trained image.

For every trained image and given test image the same process is repeated from scale and rotation normalization of the test image to the generation of abridged shape matrices, calculation and storage of similarity measure value in similarity measure vector. After all the trained images are considered for obtaining the similarity measure value, the similarity measure vector is sorted in descending order based on the similarity measure values and the class label of the given test image is determined as the class label of the trained image with which the maximum similarity value is obtained. Figure 7 depicts the overall classification procedure. The experimental results of the proposed classification method are presented in the following section. 


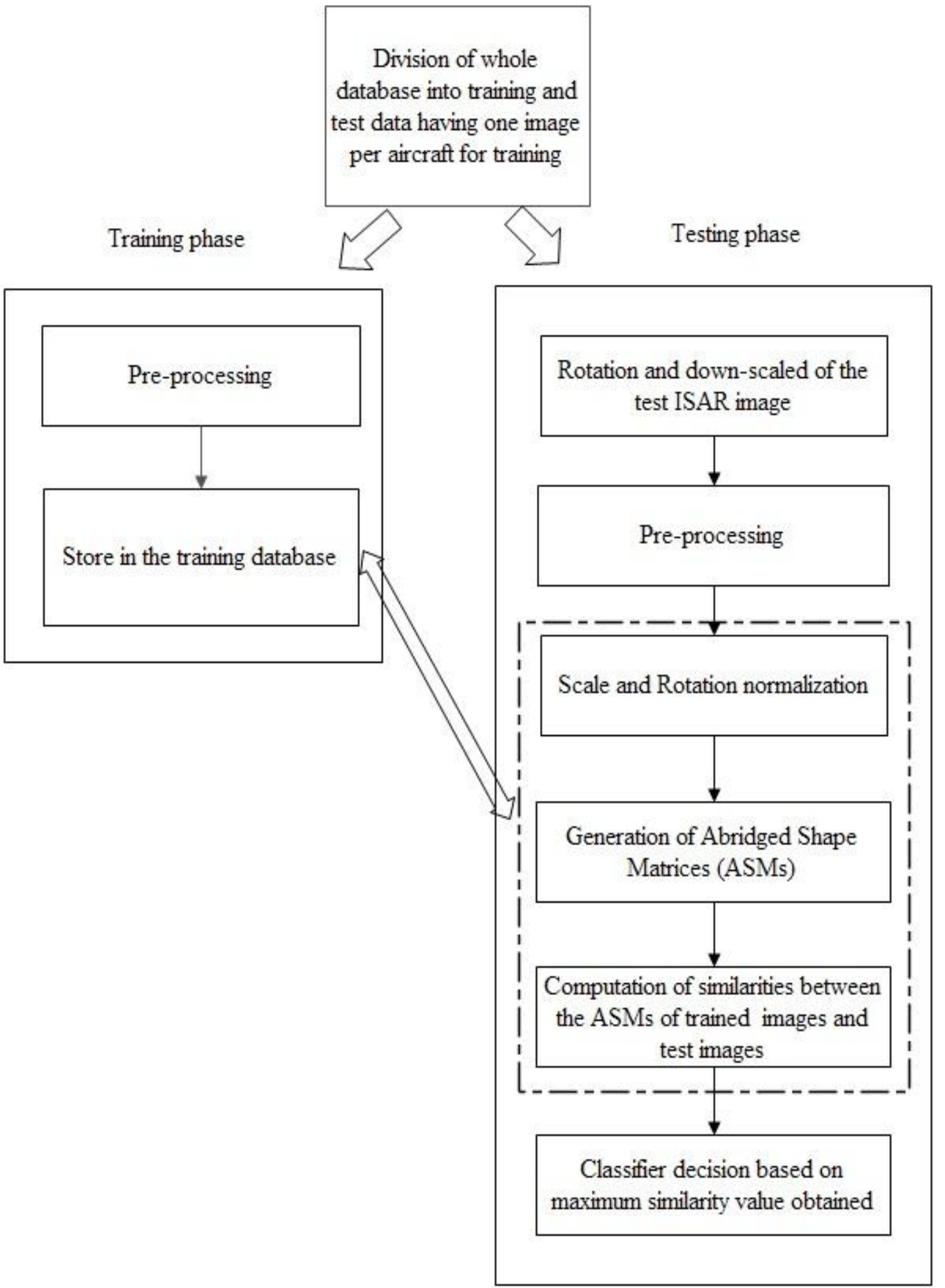

Fig. 7. Overall description of Proposed classification method 


\section{E XPERIMENTAL RESULTS}

The performance of the proposed method is compared against the polar mapping [7] and Fourier Transform Polar Mapping (FT+Polar Mapping) [10] and MECSM [11] schemes. The experimental analysis is carried out on the benchmark dataset, SynISAR (Kondaveeti (2016)). SynISAR consists of 465 ISAR images of 7 aircraft models. The input image size for the training is $300 \times 300$ in all the experiments. To carry out the experimentation, the data set consisting of 465 images is divided into training and test sets. The training data set consists of one image per each target i.e only 7 images are used as training set. The remaining 458 images are used for testing and validation. The experimental results show that, the proposed method is robust against scale and aspect angle changes in the target space.

The classification method described in [7] uses simple global threshold based noise removal procedure. The classifier decision depends on the number of samples selected in the coarse search and fine search phases. The number of sample selected in coarse and fine phases are denoted by $y$ and $\gamma$ respectively. Optimal values for $\eta$ and $\gamma$ have to be chosen manually by some experimentation to maximize the accuracy of classifier. In the experimental evaluation of this method, the authors have chosen their values as $y=30 \%$ and $\gamma=10 \%$ of the samples. The same values are used here for the evaluation of accuracy of this method.

Percentage of input images classified correctly over the number of input images given for the classification is considered as the recognition rate for the evaluation of the accuracy of the classification methods. The average value of the recognition rates obtained in three observations is considered to avoid classification errors.

Initially, the performances of the four methods are evaluated against the variation in $\mathrm{SNR}_{\mathrm{dB}}$ from $5 \mathrm{~dB}-30 \mathrm{~dB}$ in the increasing order of $5 \mathrm{~dB}$ at different aspect angles and scales of the targets in the test images. Graph given in figure 8 depicts the results obtained. The results show that the classification accuracy of the proposed method is more than $98 \%$ at various $\mathrm{SNR}_{\mathrm{dB}}$ levels. The slight performance decrease of the proposed method is due to limiting the size of the shape matrices.

When the performance variations of the four methods are observed at $\mathrm{SNR}_{\mathrm{dB}}$ is varied between $0.25 \mathrm{~dB}-1.5 \mathrm{~dB}$, the robustness of the proposed method can be seen. The classification performance of the three existing methods is decreased when the $\mathrm{SNR}_{\mathrm{dB}}$ is varied between $0.25 \mathrm{~dB}-1.5 \mathrm{~dB}$. But, the proposed method is performing outstandingly even at the low $\mathrm{SNR}_{\mathrm{dB}}$ levels, with slight variations in accuracy. Graph in the figure 9 shows the difference in the classification accuracies of four methods. The trivial noise reduction procedure and the dependency of the classifier on the selection of the $\mathrm{y}$ and $\gamma$ values adversely affected the accuracy of method [7]. 


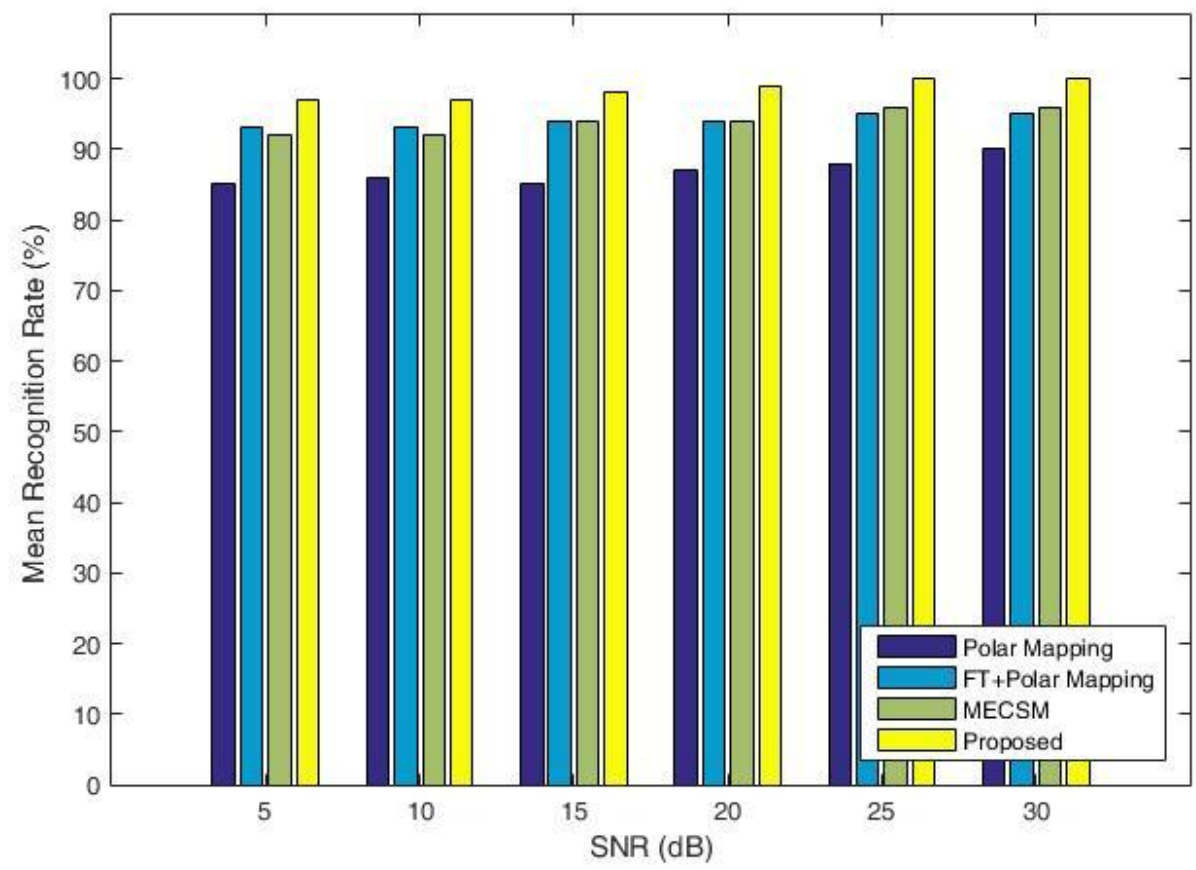

Fig. 8. Comparison of classification accuracies at $\mathrm{SNR}_{\mathrm{dB}}$ is between $5 \mathrm{~dB}-30 \mathrm{~dB}$.

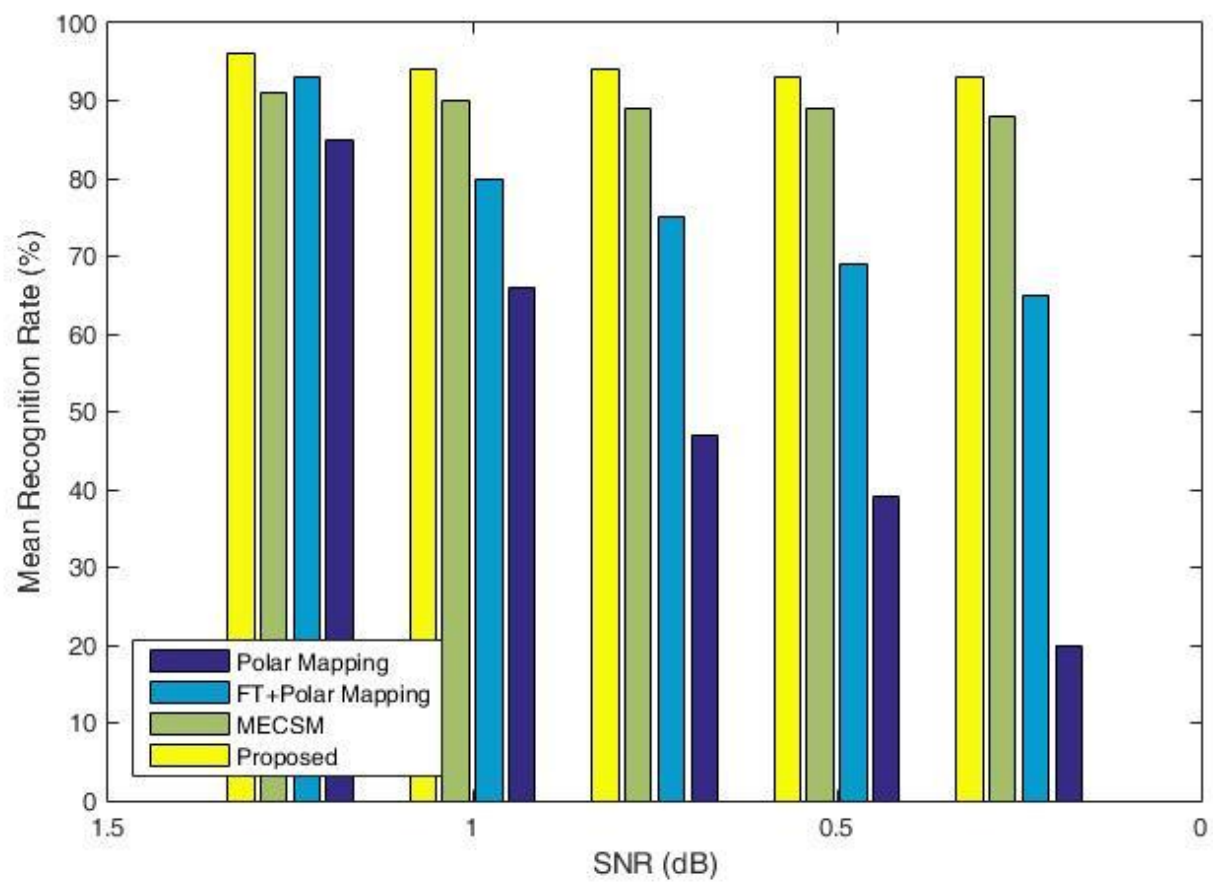

Fig. 9. Comparison of classification accuracies at $S R_{d B}$ is between $0.25 \mathrm{~dB}-1.5 \mathrm{~dB}$. 
Graph in the figure 10 depicts the classification accuracies of the four methods at various levels of blur. Finite Impulse Response (2D-FIR) filter is applied to test images to simulate blur of various orders. The proposed method is performing consistently even at higher orders of blur when compared to remaining three methods as it does not depend on the true pixel intensities of the target responses.

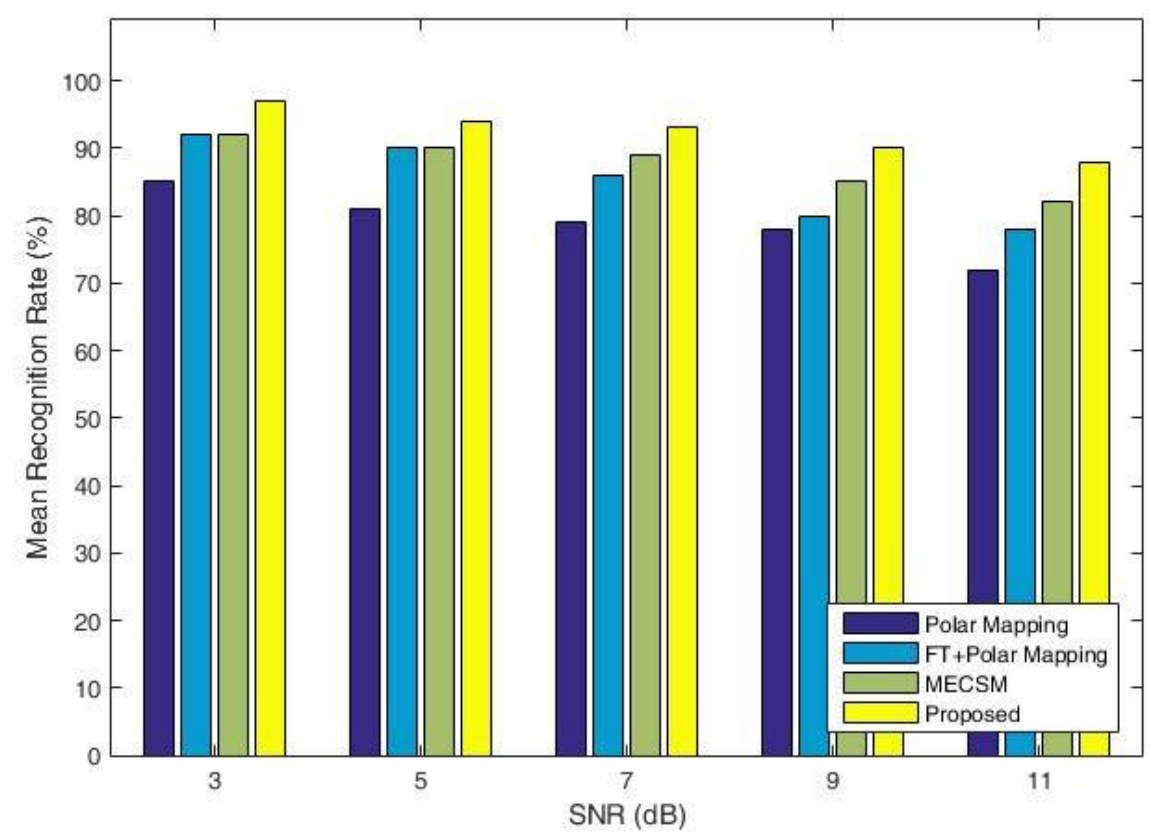

Fig. 10. Comparison of classification accuracies at various orders of blur

\section{CONCLUSION}

In this paper, a new classification method is proposed to classify aircrafts from ISAR imagery. The pre-processing module effectively segments out the target from noisy background by preserving most of the discriminative information for the classification. The proposed abridged shape matrix representation is precise and nonredundant. It avoids the unnecessary interpolations where much of the computational resources can be saved. The experimental results show that the proposed method gives good average recognition rate and robust to noise and scale changes of the targets.

\section{ACKNOWLEDGMENTS}

Authors would like to thank the anonymous reviewers for their constructive comments and reviews. 
Abridged Shape Matrix Representation for the Recognition of Aircraft Targets.. 1121

\section{REFERENCES}

[1] S. Musman, D. Kerr, and C. Bachmann, Automatic recognition of ISAR ship images, IEEE Trans. Aerospace Electron. Syst., vol. 32, no. 4, pp.13921404, Oct. 1996.

[2] C. M. Bachmann, S. A. Musman, and A. Schultz, Lateral inhibition neural networks for classification of simulated radar imagery, inProc.Int. Joint Conf. Neural Networks, vol. 2, pp. 115120, Jun. (1992).

[3] A. Maki, K.Fukui, Y.Kawawada, and M.Kiya, Automatic ship identification in isar imagery: An on-line system usig cmsm, Radar Conference, pp. 206-211, (2002)

[4] T. Kawaharay, S. Todaz, A. Mikamiz, M. Tanabez, Automatic Ship Recognition Robust Against Aspect Angle Changes and Occlusions, Radar Conference (RADAR), (2012) IEEE.

[5] M. N. Saidi, K. Daoudi, A. Khenchaf, B. Hoeltzener, and D. Aboutajdine, Automatic target recognition of aircraft models based on isar images, IEEE International Geoscience and Remote Sensing Symposium, IGARSS, Cape Town, South Africa, (2009).

[6] K. Rosenbach and J. Schiller, Identification of aircraft on the basis of 2-D radar images, in IEEE Int. Radar Conf. Record, pp.405409, May (1995).

[7] K. T. Kim , D. K. Seo and H. T. Kim, Efficient classification of ISAR images, IEEE Trans. Antennas Propagat., vol. 53, no. 5, pp.1611 -1621 (2005).

[8] E.C. Botha, Classification of aerospace targets using super-resolution ISAR images, Proc. IEEE South African Symp. on Communications and Signal Processing, pp.138 -145 (1994).

[9] F. Benedetto , F. Riganti Fulginei , A. Laudani , G. Albanese, Automatic Aircraft Target Recognition by ISAR Image Processing based on Neural Classifier

[10] Sang-Hong Park.: An Efficient Classification Method for Inverse Synthetic Aperture Radar Images, World Academy of Science, Engineerin and Technology, Vol:7 pp: , (2013)

[11] Vatsavayi, V.K., Kondaveeti, H.K.: 'Efficient ISAR image classification using MECSM representation'. Journal of King Saud University Computer and Information Sciences, 2016. http://dx.doi.org/10.1016/j.jksuci.2016.07.004

[12] Torr, P. H. S., and A. Zisserman, "MLESAC: A New Robust Estimator with Application to Estimating Image Geometry," Computer Vision and Image Understanding, 2000 
[13] J. Mateo , A. Fernandez-Caballero, Finding out general tendencies in speckle noise reduction in ultrasound images, Expert Syst. Appl. , 4 , pp: 7786 - 7797, (2009).

[14] Xinggan Zhang, Yechao Bai, ISAR Target 2D Images Pre-processing in ATR, Computer Science and Information Engineering, 2009 WRI World Congress on (Volume:6 ),March 31 2009-April 2 2009, pp.542 - 544.

[15] Gonzalez R.C, Woods R.E, Digital Image processing, Pearson Prentice Hall, 3rd edition, Eighth impression, (2012).

[16] Herbert Bay , Andreas Ess , Tinne Tuytelaars , Luc Van Gool, Speeded Up Robust Features (SURF), Computer Vision and Image Understanding, v.110 n.3, p.346-359, June, (2008).

[17] Ardeshir Goshtasby, Description and Discrimination of Planar Shapes Using Shape Matrices, IEEE Trans. on Pattern Analysis and Machine Intel., vol. PAMI-7, No.6, pp:738-743 Nov (1985)

[18] A.Taza and C.Suen, Discrimination of planar shapes using shape matrices, IEEE Trans. System, Man, and Cybernetics, vol. 19(5), pp: 1281-1289, (1989) 